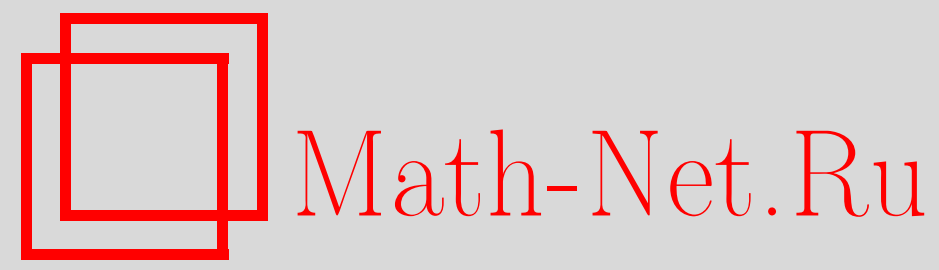

В. В. Петров, Об усиленном законе больших чисел для последовательности неотрицательных случайных величин, Теория вероятн. и ее примен., 2008, том 53, выпуск 2, 379-382

DOI: https://doi.org/10.4213/tvp2419

Использование Общероссийского математического портала Math-Net.Ru подразумевает, что вы прочитали и согласны с пользовательским соглашением

http://www.mathnet.ru/rus/agreement

Параметры загрузки:

IP : 52.6 .47 .48

26 апреля 2023 г., 12:32:54

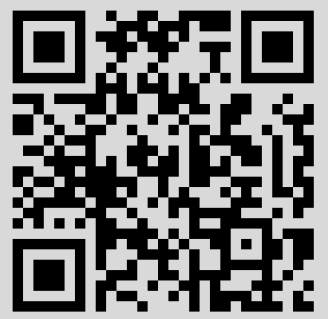


(c) 2008 r.

ПЕТРОВ В. В.*

\section{ОБ УСИЛЕННОМ ЗАКОНЕ БОЛЬШИХ ЧИСЕЛ ДЛЯ ПОСЛЕДОВАТЕЛЬНОСТИ НЕОТРИЦАТЕЛЬНЫХ СЛУЧАЙНЫХ ВЕЛИЧИН ${ }^{1)}$}

Найдены широкие достаточные условия применимости усиленного закона больших чисел к последовательности неотрицательных случайных величин с конечными дисперсиями.

Ключевые слова и фразы: усиленный закон больших чисел, последовательности неотрицательных случайных величин.

Пусть $X_{1}, X_{2}, \ldots$ - произвольная последовательность случайных величин с конечными дисперсиями. Положим $S_{n}=X_{1}+X_{2}+\cdots+X_{n}$. В силу неравенства Чебышева имеем

$$
\mathbf{P}\left\{\left|S_{n}-\mathbf{E} S_{n}\right| \geqslant n \varepsilon\right\} \leqslant(n \varepsilon)^{-2} \mathbf{D} S_{n}
$$

для любого $\varepsilon>0$. Если

$$
\mathrm{D} S_{n}=o\left(n^{2}\right) \quad(n \rightarrow \infty),
$$

то $\left(S_{n}-\mathbf{E} S_{n}\right) / n \rightarrow 0$ по вероятности. Эта форма слабого закона больших чисел была получена Марковым без каких-либо дополнительных условий независимости. Условие (2) будем называть условием Маркова. Некоторое усиление условия Маркова приводит, как показано ниже, к усиленному закону больших чисел в форме

$$
\frac{S_{n}-\mathbf{E} S_{n}}{n} \rightarrow 0 \text { п.н. }
$$

Нам понадобятся следующие обозначения. Следуя $[1$, гл. 9], будем использовать обозначение $\Psi_{c}$ для множества функций $\psi(x)$ таких, что каждая $\psi(x)$ положительна и не убывает в области $x>x_{0}$ при некотором $x_{0}$ и ряд $\sum(n \psi(n))^{-1}$ сходится. Здесь $\sum f(n)$ означает суммирование по всем целым положительным $n$, для которых значения $f(n)$ определены и неотрицательны. Значение $x_{0}$ не предполагается одним и тем же для различных функций $\psi$. Если в этом определении мы заменим слово «сходится» словом «расходится», то мы получим определение класса функций $\Psi_{d}$. Примерами функций класса $\Psi_{c}$ являются функции $x^{\alpha}$ и $(\ln x)^{1+\alpha}$ при любом $\alpha>0$. Функции $\ln x$ и $\ln x \ln \ln x$ принадлежат классу $\Psi_{d}$.

Лемма 1. Если $\psi(x) \in \Psi_{c}$, то ряд $\sum 1 / \psi\left(b^{n}\right)$ сходится для любого $b>1$.

Доказательство этой леммы опирается на следующее предложение.

Лемма 2. Пусть $\left\{a_{n}\right\}-$ последовательность неотричательньи иисел, $A_{n}=$ $a_{1}+a_{2}+\cdots+a_{n}, A_{n} \rightarrow \infty(n \rightarrow \infty)$. Тогда ряд $\sum a_{n} /\left(A_{n} \psi\left(A_{n}\right)\right)$ сходится для любой $\psi \in \Psi_{c}$.

Доказательство леммы 2 можно найти в $[1$, гл. 9]. Применяя лемму 2 к случаю $a_{k}=b^{k-1}(k \geqslant 1)$, мы приходим к утверждению леммы 1 . Другое доказательство леммы 1 можно найти в [2].

Важную роль в дальнейшем будет играть условие

$$
\mathrm{D} S_{n}=O\left(\frac{n^{2}}{\psi(n)}\right) \quad \text { для некоторой функции } \quad \psi \in \Psi_{c} .
$$

* С.-Петербургский государственный университет, математико-механический факультет, Старый Петергоф, 198504 С.-Петербург, Россия.

1) Работа выполнена при поддержке Российского фонда фундаментальных исследований (грант № 07-01-00688). 
Лемма 3. Пусть $\left\{X_{n}\right\}$ - последовательность независимьх случайньх величин с конечньми дисперсиями. Если выполнено условие (4), то имеет место соотношение (3).

Это предложение доказано в [3] с помощью полученных там оценок роста сумм независимых случайных величин почти наверное (см. также $[1$, гл. 9 , теорема 26]). Там же было показано, что условие (4) в лемме 3 нельзя ослабить, потребовав вместо него выполнение содержащегося в (4) равенства для некоторой функции $\psi \in \Psi_{d}$. Заметим, что любая функция $\psi \in \Psi_{d}$ растет медленнее, чем любая функция $\psi \in \Psi_{c}$, далее, $\psi(n) \rightarrow \infty(n \rightarrow \infty)$ для любой $\psi \in \Psi_{c}$.

Мы приведем более простое по сравнению с [3] доказательство леммы 3. По неравенству Чебышева имеем

$$
\mathbf{P}\left\{\left|S_{2^{n}}-\mathbf{E} S_{2^{n}}\right| \geqslant 2^{n} \varepsilon\right\} \leqslant\left(2^{n} \varepsilon\right)^{-2} \mathbf{D} S_{2^{n}}
$$

для любого $\varepsilon>0$. В силу условия (4) получим

$$
\mathbf{P}\left\{\left|S_{2^{n}}-\mathbf{E} S_{2^{n}}\right| \geqslant 2^{n} \varepsilon\right\} \leqslant C\left(\varepsilon^{2} \psi\left(2^{n}\right)\right)^{-1}
$$

при всех достаточно больших $n$, где $C$ - постоянная. Принимая во внимание условие $\psi \in \Psi_{c}$ и лемму 1 , находим, что

$$
\sum_{n=1}^{\infty} \mathbf{P}\left\{\left|S_{2^{n}}-\mathbf{E} S_{2^{n}}\right| \geqslant 2^{n} \varepsilon\right\}<\infty
$$

для любого $\varepsilon>0$. По лемме Бореля-Кантелли $\mathbf{P}\left\{\left|S_{2^{n}}-\mathbf{E} S_{2^{n}}\right| \geqslant 2^{n} \varepsilon\right.$ бесконечно часто $\}=0$ для любого $\varepsilon>0$ и, следовательно,

$$
2^{-n}\left(S_{2^{n}}-\mathbf{E} S_{2^{n}}\right) \rightarrow 0 \text { п.н. }
$$

Заметим, что до сих пор предположение о независимости не было использовано. Применим теперь один результат Каваты (см., например, $[1$, с. 350]), в силу которого для последовательности независимых случайных величин $\left\{X_{n}\right\}$, удовлетворяющей условиям $S_{n} / n \rightarrow 0$ по вероятности и $\sum_{n=1}^{\infty} \mathbf{P}\left\{\left|S_{2^{n}}\right| \geqslant 2^{n} \varepsilon\right\}<\infty$ для любого $\varepsilon>0$, выполнено соотношение $S_{n} / n \rightarrow 0$ п.н. Тогда получим (3) как следствие (5). Лемма 3 доказана.

В дальнейшем мы не делаем предположения о независимости случайных величин из исходной последовательности.

Побочным продуктом доказательства леммы 3 является следуюшее предложение.

Лемма 4. Пусть $\left\{X_{n}\right\}$ - последовательность случайньх величин с конечньми дисперсиями. Если выполнено условие (4), то имеют место соотношения (6) и (5) для любого $\varepsilon>0$.

Перейдем теперь к формулировке основных результатов работы.

Теорема 1. Пусть $\left\{X_{n}\right\}$ - последовательность неотричательных случайных величин с конечньми дисперсиями. Положим

$$
S_{n, m}=\sum_{i=m+1}^{n} X_{i}
$$

длs чельх $m \geqslant 0 u n>m$,

$$
S_{n}=S_{n, 0}=\sum_{i=1}^{n} X_{i} .
$$

Пусть выполнены условия (4) $и$

$$
\mathbf{E} S_{n, m} \leqslant C(n-m)
$$

для всех достаточно больиих $n-m$, аде $C$ - постоянная. Тогда имеет место соотношение (3).

Д о к а з а т е л с т в о. Пусть $b>1, k_{n}=\left[b^{n}\right]$. По неравенству Чебышева имеем

$$
\sum_{n=1}^{\infty} \mathbf{P}\left\{\left|S_{k_{n}}-\mathbf{E} S_{k_{n}}\right| \geqslant k_{n} \varepsilon\right\} \leqslant \varepsilon^{-2} \sum_{n=1}^{\infty} \frac{\mathbf{D} S_{k_{n}}}{k_{n}^{2}}
$$


для любого $\varepsilon>0$. Используя условие (4) и лемму 1 , получим

$$
\sum_{n=1}^{\infty} \frac{\mathrm{D} S_{k_{n}}}{k_{n}^{2}} \leqslant C \sum_{n=1}^{\infty} \frac{1}{\psi\left(k_{n}\right)}<\infty .
$$

Применение леммы Бореля-Кантелли приводит к соотношению

$$
\frac{S_{k_{n}}-\mathbf{E} S_{k_{n}}}{k_{n}} \rightarrow 0 \text { п.н. }
$$

Если $k_{n} \leqslant k<k_{n+1}$, то с учетом неотрицательности случайных величин из исходной последовательности получаем

$$
\begin{aligned}
& \frac{S_{k}-\mathbf{E} S_{k}}{k} \leqslant \frac{\left|S_{k_{n+1}}-\mathbf{E} S_{k_{n+1}}\right|}{k_{n+1}} \frac{k_{n+1}}{k_{n}}+\frac{\mathbf{E} S_{k_{n+1}}-\mathbf{E} S_{k_{n}}}{k_{n}}, \\
& \frac{S_{k}-\mathbf{E} S_{k}}{k} \geqslant \frac{\left|S_{k_{n}}-\mathbf{E} S_{k_{n}}\right|}{k_{n}} \frac{k_{n}}{k_{n+1}}-\frac{\mathbf{E} S_{k_{n+1}}-\mathbf{E} S_{k_{n}}}{k_{n+1}} .
\end{aligned}
$$

Вследствие (9) первые слагаемые в правых частях (10) и (11) сходятся к нулю почти наверное. Второе слагаемое в правой части (10) при всех достаточно больших $n$ не превосходит $C\left(k_{n+1}-k_{n}\right) / k_{n}$ в силу условия (7). Такая же оценка справедлива и для дроби во втором слагаемом (11), так как $k_{n} \leqslant k_{n+1}$. Поэтому $\lim \sup _{k \rightarrow \infty} \mid S_{k}-$ $\mathbf{E} S_{k} \mid / k \leqslant C(b-1)$ п.н.

Правая часть последнего неравенства может быть сделана сколь угодно малой за счет выбора числа $b$ достаточно близким к 1. Отсюда следует соотношение (3).

Покажем теперь, что в теореме 1 нельзя заменить условие (4) более слабым условием

$$
\mathrm{D} S_{n}=O\left(\frac{n^{2}}{\psi(n)}\right) \quad \text { для некоторой функции } \quad \psi \in \Psi_{d} .
$$

Теорема 2. Для любой функиии $\psi \in \Psi_{d}$ такой, что $n / \psi(n)$ не убьвает в области $n>n_{0}$ при некотором $n_{0}$, существует последовательность неотричательных попарно независимых случайных величин с двумя значениями, для которой выполнены условия (7) и (12), но соотношение (3) не имеет места.

Д ок а з а т е л в с т в о. Пусть $\psi \in \Psi_{d}$, а $n / \psi(n)$ не убывает. Рассмотрим последовательность попарно независимых случайных величин $\left\{X_{n}\right\}$ такую, что при $n>N$ случайная величина $X_{n}$ имеет значения 0 и $n$ с вероятностями $1-1 /(n \psi(n))$ и $1 /(n \psi(n))$ соответственно. Номер $N$ выберем таким образом, что $N \psi(N)>1$ и $N>n_{0}$. Пусть при $n \leqslant N$ случайная величина $X_{n}$ имеет значения 0 и 2 , каждому из которых соответствует вероятность $\frac{1}{2}$. Тогда

$$
\begin{aligned}
& \mathbf{E} X_{n}=1, \quad \mathbf{E} X_{n}^{2}=2 \quad(n \leqslant N), \\
& \mathbf{E} X_{n}=\frac{1}{\psi(n)}, \quad \mathbf{E} X_{n}^{2}=\frac{n}{\psi(n)}, \quad \mathbf{E} S_{n}=N+\sum_{k=N+1}^{n} \frac{1}{\psi(k)} \quad(n>N) .
\end{aligned}
$$

Поэтому

$$
\mathbf{E} S_{n}-\mathbf{E} S_{m}=\sum_{k=m+1}^{n} \frac{1}{\psi(k)} \quad \text { при } \quad n>m>N,
$$

так что условие (7) выполнено. Далее, вследствие попарной независимости рассматриваемых случайных величин имеем

$$
\mathbf{D} S_{n}=\sum_{k=1}^{n} \mathbf{D} X_{k} \leqslant \sum_{k=1}^{n} \mathbf{E} X_{k}^{2}=2 N+\sum_{k=N+1}^{n} \frac{k}{\psi(k)} \leqslant 2 N+\frac{(n-N) n}{\psi(n)}
$$

при $n>N$ с учетом условия неубывания функции $n / \psi(n)$. Отсюда следует, что условие (12) выполнено. Покажем теперь, что соотношение (3) не выполнено.

Если бы (3) было выполнено, то в силу (3) и очевидного тождества

$$
\frac{S_{n}-\mathbf{E} S_{n}}{n}=\frac{S_{n-1}-\mathbf{E} S_{n-1}}{n-1} \frac{n-1}{n}+\frac{X_{n}-\mathbf{E} X_{n}}{n}
$$


мы имели бы соотношение $\left(X_{n}-\mathbf{E} X_{n}\right) / n \rightarrow 0$ п.н., которое противоречит равенству $\mathbf{P}\left\{X_{n}=n\right.$ бесконечно часто $\}=1$, вытекающему из условия $\psi \in \Psi_{d}$, равенств

$$
\sum \mathbf{P}\left\{X_{n}=n\right\}=\sum \frac{1}{n \psi(n)}=\infty
$$

и леммы Бореля-Кантеллли в формулировке Эрдёша-Реньи.

Приведем одно следствие теорем 1 и 2 . Если $\left\{X_{n}\right\}$ - последовательность неотрицательных случайных величин с конечными дисперсиями, удовлетворяющая условию (7), то условие $\mathrm{D} S_{n}=O\left(n^{2} /(\ln n)^{1+\delta}\right)$ для некоторого $\delta>0$ достаточно для справедливости усиленного закона больших чисел в форме (3). Если же выполнено несколько более слабое условие $\mathrm{D} S_{n}=O\left(n^{2} / \ln n\right)$ или даже условие $\mathbf{D} S_{n}=$ $O(n /(\ln n \ln \ln n))$, то соотношение (3) может не выполняться.

Для последовательности неотрицательных случайных величин с конечными дисперсиями иные достаточные для (3) условия были найдены Н. Этемади [4] (см. также $[5$, с. 244$])$, эти условия включают равномерную ограниченность математических ожиданий рассматриваемых случайных величин и неположительность коэффициентов корреляции между любыми случайными величинами с различными индексами.

\section{СПИСОК ЛИТЕРАТУРЫ}

1. Петров B. В. Суммы независимых случайных величин. М.: Наука, 1972, 414 с.

2. Петров $B$. $B$. Некоторые оценки для сумм зависимых случайных величин, имеюшие место почти наверное. - Записки науч. сем. ЛОМИ, 1976, т. 55, с. 113-116.

3. Петров В. В. Об усиленном законе больших чисел. - Теория вероятн. и ее примен., 1969 , т. 14 , в. 2 , с. $193-202$.

4. Etemadi $N$. On the law of large numbers for nonnegative random variables. - J. Multivariate Anal., 1983, v. 13, № 1, p. 187-193.

5. Петров В. В. Предельные теоремы для сумм независимых случайных величин. М.: Наука, 1987, 317 c.

Поступила в редакцию

6.II.2008

(C) $2008 \mathrm{r}$.

\section{РОЗОВСКИЙ Л. В.* О ГАУССОВОЙ МЕРЕ ШАРОВ В ГИЛЬБЕРТОВОМ ПРОСТРАНСТВЕ ${ }^{1)}$}

Пусть $X$ - центрированный гауссов случайный вектор со значениями в сепарабельном гильбертовом пространстве $H$, и пусть $a \in H$. Мы изучаем с помошью преобразования Лапласа поведение плотности и функции распределения шара $\|X-a\|^{2}$; получены результаты с оптимальной оценкой. Наш подход связан с «локальными предельными теоремами».

Ключевые слова и фразы: малые шары, гауссова мера, гильбертово пространство, преобразование Лапласа.

* С.-Петербургская химико-фармацевтическая академия, кафедра высшей математики, ул. проф. Попова, 14, 197376 С.-Петербург, Россия; e-mail: 1_rozovsky@mail.ru

1) Работа выполнена при поддержке Министерства образования РФ (грант Е02-1.0-219), РФФИ (грант 02-01-01099) и программы «Ведушие научные школы» (грант НШ-2258.2003.1). 